\title{
Filling Driven by Contour Marching
}

\author{
Gilles Mathieu \\ LISSE \\ Centre SIMADE, Ecole des Mines \\ 158 cours Fauriel 42023 Saint-Etienne Cedex 2 France \\ tel 77420175 - fax 77426666 \\ email mathieu@emse.fr
}

\begin{abstract}
A filling algorithm is proposed that works in a matrix of pixels. It proceeds by spans and is directed by contour marching, a contour being viewed as a circular list of linels. This technique has 3D applications: it is used in traversing the surfels graph of a voxelized object.
\end{abstract}

\section{Introduction}

Two-dimensional filling or coloring problems have received many solutions in the past. First methods were influenced by vector graphics technology and thus by continuous methods. They are inspired by polygon scan-conversion with ordered active edges list [FVFH90].

Among discrete methods, a second kind of approach aims at adapting the parity check technique to raster memory. Pavlidis has proposed various algorithms to solve what he calls undersampling problems (different boundary points being merged in a single pixel, diagonal contact between pixels) [Pav79].

An important family is the one of connexity methods. A seed pixel is supposed to be known. Depending on wether the transitive closure is done on pixels with the same value as the seed, or on those of value distinct from that of a boundary pixel, a distinction is made between flood and boundary techniques [FVFH90, p.979] (Hégron used the term coloring, in contrast to flling [Heg85, p.67]). Coloring better fits to an interactive use, because the automatic determination of the seed is tricky. The child's coloring book metaphor was first proposed by Lieberman [Lie78].

Most of the works consider spans which are limited by two boundary pixels and do not contain any pixel with the new color. Smith avoids redundant contiguous span explorations [Smi79]. Most of the methods also resort to a stack for the coloring fronts. At any time, the already colored part is limited by the object boundary, the current span and these fronts.

Shani emphasized that contour filling reduces to graph traversal [Sha80]. In his wake, Pavlidis unified parity check and filling using boundary line adjacency graph [Pav81]. Nodes in this graph of degree greater than 1 are decisive, because they correspond to vertical boundary extremums.

Finally, Tang et Lien analyse the Freeman code of the contour to fill [TL88].

The proposed filling method proceeds by spans. Its originality lies in the fact that it considers contours as circular lists of linels rather than pixels. Moreover, the will to minimize pixel map accesses has guided its elaboration. Finally, unlike the previous approaches, it is recursive and the processing of spans is directed by contour marching. 
The next section gives the suitable definitions: some usual discrete topological notions, and definitions peculiar to our method. Section 3 is an informal and illustrated description of the algorithm. Section 4 discusses about complexity and advantages of our approach with respect to other techniques. Finally, the last section outlines the application of this filling algorithm to surface traversal of voxelized objects.

\section{Definitions}

\subsection{Discrete topology}

We review some of the definitions given by Kong and Rosenfeld in [KR89]. Let us consider a binary bidimensional matrix of pixels. Each pixel is associated with a lattice point, that is a point of the plane with integer coordinates. A lattice point associated with a pixel that has value 1 (resp. 0) is called black (resp. white).

A 3D (resp. 2D, 1D) unit cell is a cube (resp. a square, a segment) whose linear length is 1 and whose vertices are lattice points. A OD unit cell is a single lattice point. In a 3D space, following the terminology adopted by Françon, we will respectively speak of voxels, surfels, linels and pointels, by analogy with pixels, unit cells in a $2 \mathrm{D}$ space [Fra95].

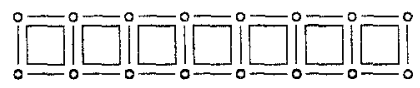

Fig. 1. Pixels (squares), linels (segments) and pointels (circles)

A digital picture is a quadruple $\mathcal{I}=(V, m, n, B)$ where $B \subseteq V=\mathbb{Z}^{2}$ is the black points set. $(m, n)=(8,4)$ or $(4,8)$. Two black points are said to be adjacent if they are $m$-adjacent. For any other pair of points $n$-adjacency is used.

A set $S$ of black and/or white points in a digital picture is connected if $S$ cannot be partitioned into two non adjacent subsets. A component of a set of black and/or white points $S$ is a non-empty maximal connected subset of $S$ (i.e. it is not adjacent to any other point in $S$ ). A component of the set of all black (resp. white) points of a digital picture is called a black component (resp. white component). The digital picture is supposed to be finite. So there is a unique infinite white component, called the background. Let $X$ and $Y$ be two sets of points in a digital picture $(V, m, n, B), X$ being connected. Then $X$ is said to surround $Y$ if each point in $Y$ is contained in a finite component of $V-X$. In 2D, a white component adjacent to a black component $C$ and surrounded by it is called a cavity of $C$.

A black point is a border point if it is adjacent to one or more white points. Otherwise, it is called an interior point. The border (resp. interior) of a black component $C$ of $\mathcal{I}$ is the set of every border (resp. interior) points of $C$.

We will use the classical notions of $x y$-convexity and $x y$-concavity. For a precise definition, we refer to [MF82] or to the automat given in [Mat95].

\subsection{Span filling directed by contour marching}

Let us consider a digital picture $\mathcal{I}=(V, 8,4, B)$. Let us suppose that $B$ has a unique component we call object $O$. This objet will possibly have holes: $V-B$ may have many components. 
By contour, we mean the set of closed curves of border linels, that is circular lists of linels separating $O$ from $\bar{O}$. Between $O$ and the background stands an exterior contour. It is traced clockwise. Between $O$ and the possible cavities stand interior contours. They are traversed counterclockwise. The fonction march_contour () will actually involve a contour marching algorithm [CM91]. But when the circular chaining of linels is built, it is enough to follow the links. A variable current contains a contour linel. It is initialized with an horizontal upper linel of a black pixel, taken in the first non empty scan line. Interior exploration is done by means of spans. A span is a maximal connected set of black points on a line of the picture. Thus spans are horizontal. They will systematically be explored from left to right. From a vertical left linel, explore_span() progresses inside the object and returns the first right linel encountered. Each span is caracterized by two vertical border linels which delimit it. In the sequel, we denote them by their two begin and end fields. Ends of the current span will be given by current and end. Taking into account only vertical border linels, we need fonctions is_vertical () and is_left(). Fonction is_exterior() is useful as well. Figure 2a shows the contour of an simple object. Figure $2 \mathrm{~b}$ shows its two spans. Useful linels are numbered.
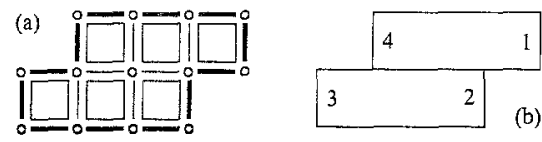

Fig. 2. Contour and spans

The algorithm uses a global table to mark linels. Initially, a linel mark is null, what can be tested by function is_new(). Function color() treats a span. It either assigns the color of the span pixels or it stores its ending linels for later use. In each case, the latter are marked such that is_colored () avoids coloring them again.

The method makes use of stacks and so has at its disposal the traditionnal primitives create_stack (), empty_stack (), push (), top () et pop (). Some stacks are sometimes accessed as queues, so operations enqueue(), bottom () and dequeue () have been added.

\section{Description of the algorithm}

We will present the algorithm in an informal way by showing its execution on some examples. They hare an increasing complexity ( $x y$-convex object without cavity, object without cavity, object with cavities), so this allows an "incremental" understanding of the spirit of our method and of the data structures role. For a more formal approach, the reader is refered to the pseudo-code given in appendix. Throughout the text, $i_{j}$ refers to instruction number $j$. The main loop presents six cases of iteration end. The first three ones work for a left current linel and the last three ones for a right current linel. Those cases are indicated as comments in the pseudo-code and in the last column in the tables showing the stacks states. In the text, $l_{i}$ refers to the line of the table for which current is $i$. Significant elements of that line are in bold. Finally, in the figures, circled numbers give the order in which spans are colored.

The general methodology consists in marking each linel while marching along the contour, clockwise for the exterior contour, counterclockwise for an interior one. For a 
vertical left met linel, a span exploration is carried out. For a vertical linel encountered again, or for a right linel, instead of exploring a span we retrieve it from one of the stacks explored (local) or remainder (global). Another global stack linels stores already encountered but not yet belonging to a colored span. The algorithm is called recursively for each discovery of an interior contour. The detection of a potential cavity happens when the exploration ends up at a never encountered linel. Confirmation is provided only farther on the current contour marching, so a last data structure is needed. Local variable discovery stores a span that may lead to a new contour. But let us explain more deeply the whole process by means of examples.

\section{$3.1 \quad X y$-convex object without cavity}

\begin{tabular}{|c|c|c|c|c|l|}
\hline cur. & end & disc. & linels & explored & case \\
\hline 1 & - & - & $\mathbf{1}$ & $\emptyset$ & 6 \\
2 & - & - & $\mathbf{2}$ & $\emptyset$ & 6 \\
3 & $\mathbf{2}$ & - & 1 & $\emptyset$ & $1: 3-2$ \\
4 & $\mathbf{1}$ & - & $\emptyset$ & $\emptyset$ & $1: 4-1$ \\
\hline
\end{tabular}

Table 1. Execution for an xy-convex objet without cavity (see fig. 2)

The main loop consists in marching the whole exterieur contour $\left(i_{1}, i_{7}, i_{8}, i_{9}\right)$, taking into account only vertical linels $\left(i_{10}, i_{11}\right)$. Each new encountered linel is marked with the current contour index, here $n_{-}$contour $=1\left(i_{7}\right)$. According to the choices given above for the initial linel and the directions of marching, right linels come up first. They are pushed into the global stack linels ( $i_{31}$, case $6, l_{1}$ and $l_{2}$ table 1 ). After that, left linels are found $\left(i_{12}\right)$. A span exploration is done from the beginning of each of them $\left(i_{13}\right)$. It necessarily ends up at the linel on top of the stack $\left(i_{15}\right)$. The latter is then popped and the span colored $\left(i_{16}\right.$, case $1, l_{3}$ and $l_{4}$ table 1$)$. In this first coloring case, the left vertical linel of the span is always encountered after its right one.

\subsection{Object without cavity}

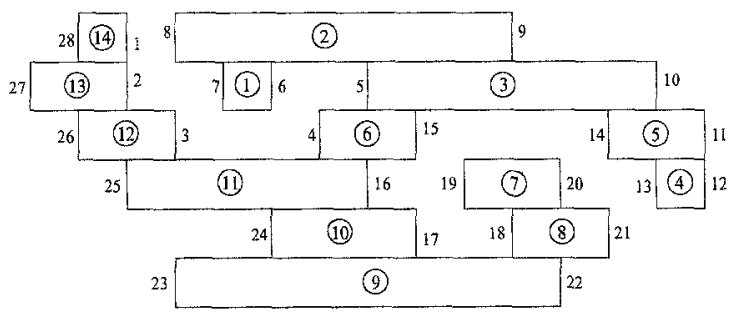

In the presence of $x y$-concavities, an exploration beginning at a left linel may now not end up at the linel on top of linels anymore $\left(l_{4}\right.$ table $2:$ top 3 , end 15). This left 
linel is then pushed into Iinels ( $\left.2_{20}\right)$. A stack explored, local to the call, stores the span just explored $\left(i_{26}\right.$, case 3$)$. For a right linel, we examine the exploration stored on top of explored, by means of already_explored ()$\left(i_{23}\right.$, end between parentheses in the tables). If this span end do not correspond to the current linel, its begin is simply pushed into linels ( $i_{31}$, case $6, l_{11}$ table 2 ). In the opposite case, the span is colored and the two stacks popped ( $i_{30}$, case $5, l_{9}$ table 2 ). In this second coloring case, the span left linel is always encountered before its right one.

\begin{tabular}{|c|c|c|c|c|c|}
\hline cur. & end & disc. & linels & explored & case \\
\hline 1 & - & - & 1 & $\emptyset$ & 6 \\
\hline 2 & - & - & 2 & $\emptyset$ & 6 \\
\hline 3 & - & - & $\ldots 3$ & $\emptyset$ & 6 \\
\hline 4 & 15 & $4-15$ & $\ldots 4$ & $4-15$ & 3 \\
\hline 5 & 10 & $4-15$ & 5 & $4-155-10$ & 3 \\
\hline 6 & (10) & $4-15$ & $\ldots 6$ & $4-155-10$ & 6 \\
\hline 7 & 6 & $4-15$ & $\ldots 5$ & $4-155-10$ & $1: 7-6$ \\
\hline 8 & 9 & $4-15$ & $\ldots 8$ & $4-15 \quad 5-10 \quad 8-9$ & 3 \\
\hline 9 & (9) & $4-15$ & $\ldots 5$ & $4-155-10$ & $5: 8-9$ \\
\hline 10 & (10) & $4-15$ & $\ldots 4$ & $4-15$ & $5: 5-10$ \\
\hline 11 & (15) & $4-15$ & $\ldots 11$ & $4-15$ & 6 \\
\hline 12 & $(15)$ & $4-15$ & $\ldots 12$ & $4-15$ & 6 \\
\hline 13 & 12 & $4-15$ & $\ldots .11$ & $4-15$ & $1: 13-12$ \\
\hline 14 & 11 & $4-15$ & $\ldots 4$ & $4-15$ & $1: 14-11$ \\
\hline 15 & (15) & - & $\ldots 3$ & $\emptyset$ & $5: 4-15$ \\
\hline 16 & - & - & $\ldots 16$ & $\theta$ & 6 \\
\hline 17 & - & - & $\ldots 17$ & $\theta$ & 6 \\
\hline 18 & 21 & $18-21$ & $\ldots 18$ & $18-21$ & 3 \\
\hline 19 & 20 & $18-21$ & $\ldots \ldots 19$ & $18-21 \quad 19-20$ & 3 \\
\hline 20 & (20) & $18-21$ & $\ldots 18$ & $18-21$ & $5: 19-20$ \\
\hline 21 & (21) & - & $\ldots 17$ & $\emptyset$ & $5: 18-21$ \\
\hline 22 & - & - & $\ldots 22$ & $\emptyset$ & 6 \\
\hline 23 & 22 & - & $\ldots 17$ & $\emptyset$ & $1: 23-22$ \\
\hline 24 & 17 & - & $\ldots 16$ & 10 & $1: 24-17$ \\
\hline 25 & 16 & - &. .3 & $\emptyset$ & $1: 25-16$ \\
\hline 26 & 3 & - & .2 & $\emptyset$ & $1: 26-3$ \\
\hline 27 & 2 & - & 1 & $\emptyset$ & $1: 27-2$ \\
\hline 28 & 1 & - & $\emptyset$ & $\emptyset$ & $1: 28-1$ \\
\hline
\end{tabular}

Table 2. Execution for an objet without cavity

\subsection{Object with cavities}

When explored is empty, a span exploration ending up at a linel different from linels top possibly means the discovery of a cavity. So this span is stored in a variable discovery $\left(i_{21}, i_{23}, i_{25}\right)$, local to each recursive call. Sometimes, it is a false alarm: linel discovery->end belongs to the contour. It will be encountered later and discovery canceled (figure $3 a$, table 2). In other cases, we actually deal with a cavity (figure 3b, table 3). While discovery is not assigned, explored spans are pushed in global stack remainder $\left(i_{26}\right.$, case $3, l_{14}$ table 3$)$. 


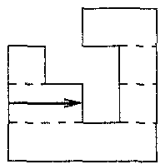

(a)

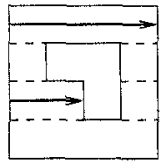

(b)

Fig. 3. Cavities : (a) false discovery (b) true one

The assignation of discovery is not sufficient to detect a cavity. Three situations are possible, as illustrated on figure 4 . In the first one, the end of the exploration is different from the linel on top of linels and it has mark 1 of the exterior contour. Then it is certain that a cavity lies under this last explored span or on the right of the current contour $\left(i_{17}, l_{14}\right.$ table 3$)$. In the example of figure $4 \mathrm{a}$, span 2 confirms the presence under it of a cavity discovered with span 1 . On figure $4 \mathrm{~d}$, span 8 confirms the presence on the right of the current contour of a cavity discovered with span 6 .

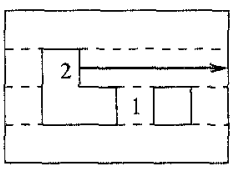

(a)

(b)

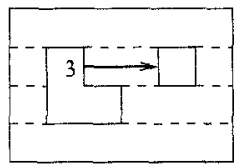

(c)
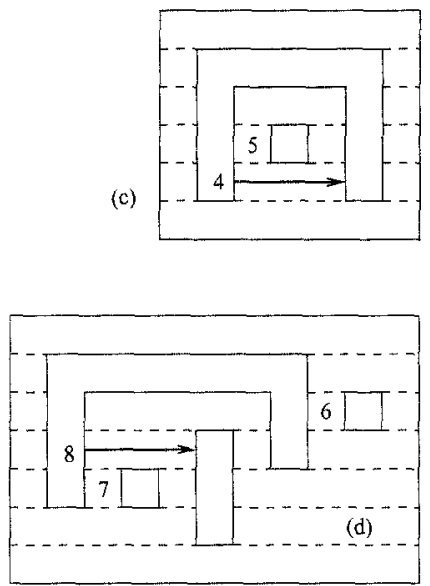

Fig. 4. Different cases for recursion

In the second situation, the current contour marching completes without any exploration having ended up at an exterior linel. The span discovery leads in this case to a cavity on the right of the current contour $\left(i_{8}, i_{9}, l_{20}\right.$ et $l_{32}$ table 3$)$. In the example of figure $4 \mathrm{~b}$, the discovery made with span 3 is confirmed only after completion of the contour marching.

Finally, in the last situation, the current interior contour marching reaches discovery $\rightarrow$ end. Thus it is certain that a cavity lies above the discovery span $\left(i_{27}\right)$. In that case, the span that actually leads to the cavity stands in explored after discovery. It is determined by fonction actual_dec(), that also transfers into remainder spans what is popped from explored. On figure $4 c$, span 4 has been considered as a discovery, but the true discovery span is 5 . 


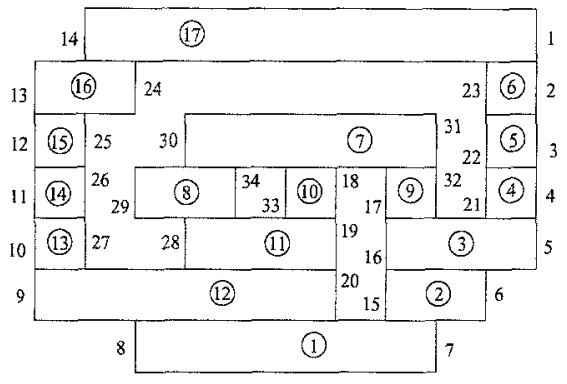

\begin{tabular}{|c|c|c|c|c|c|c|c|}
\hline cur. & end & disc & linels & explored & & remainder & case \\
\hline : & & : & & & & & \\
\hline 13 & 24 & - & $\ldots \ldots$ & $9-20 \quad 10-27 \quad 11-26$ & $\begin{array}{lll}12-25 & 13-24\end{array}$ & $\varnothing$ & 3 \\
\hline 14 & 1 & - & 13 & $\begin{array}{lllll}9-20 & 10-27 & 11-26\end{array}$ & $6 \begin{array}{lll}12-25 & 13-24\end{array}$ & $14-1$ & 2 \\
\hline 15 & 6 & - & $\ldots 5$ & \begin{tabular}{|l|l|} 
& $\phi$
\end{tabular} & & $14-1$ & $1: 15-6$ \\
\hline 16 & 5 & - & $\ldots 4$ & $\emptyset$ & & $14-1$ & $1: 16-5$ \\
\hline 17 & 32 & $\mid 17-32$ & $\ldots 17$ & $17-32$ & & 14-1 & 3 \\
\hline 18 & (32) & $17-32$ & $\ldots 18$ & $17-32$ & & $14-1$ & 6 \\
\hline 19 & (32) & $\mid 7-32$ & $\ldots \ldots 19$ & $17-32$ & & 14-1 & 6 \\
\hline 20 & (32) & $|17-32|$ & $\ldots \ldots 20$ & $17-32$ & & 14-1 & 6 \\
\hline 21 & 4 & - & 3 & & $\emptyset$ & $14-1$ & $1: 21-4$ \\
\hline 22 & 3 & - & 2 & & $\emptyset$ & $14-1$ & $1: 22-3$ \\
\hline 23 & 2 & - & 1 & & $\emptyset$ & $14-1$ & $1: 23-2$ \\
\hline 24 & - & - & 24 & & $\emptyset$ & 14-1 & 6 \\
\hline 25 & - & - & 25 & & $\emptyset$ & $\mid 14-1$ & 6 \\
\hline 26 & - & - & $\ldots 26$ & & $\emptyset$ & $14-1$ & 6 \\
\hline 27 & - & - & $\ldots 27$ & & $\emptyset$ & $14-1$ & 6 \\
\hline 28 & 19 & - & $\ldots 28$ & & $\theta$ & $14-128-19$ & 3 \\
\hline 29 & 34 & $29-34$ & $\ldots \ldots 29$ & & $29-34$ & $14-128-19$ & 3 \\
\hline 30 & 31 & $29-34$ & $\ldots \ldots 30$ & & $29-3430-31$ & $14-128-19$ & 3 \\
\hline 31 & $(31)$ & $29-34$ & $\ldots \ldots 29$ & & $29-34$ & $14-128-19$ & $5: 30-31$ \\
\hline 32 & (34) & $29-34$ & $\ldots \ldots 32$ & & $29-34$ & $14-128-19$ & 6 \\
\hline 33 & 18 & - & $\ldots \ldots \mathbf{3 3}$ & & $\emptyset$ & $\mid 14-1 \quad 28-19 \quad 33-18$ & 3 \\
\hline 34 & - & - & $\ldots \ldots 34$ & & $\emptyset$ & $\mid \begin{array}{llll}14-1 & 28-19 & 33-18\end{array}$ & 6 \\
\hline (29) & (34) & - & $\ldots \ldots 33$ & & $\emptyset$ & $14-128-1933-18$ & $1: 29-34$ \\
\hline 32 & - & - & $\ldots \ldots 32$ & & $\emptyset$ & $\mid 14-128-1933-18$ & 6 \\
\hline$(17)$ & $(32)$ & 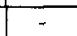 & $\ldots \ldots 33$ & $\phi$ & & $\mid 14-128-1.933-18$ & $1: \overline{17-32}$ \\
\hline 18 & - & - & $\ldots \ldots 28$ & $\emptyset$ & & $14-128-19$ & $5: 33-18$ \\
\hline 19 & - & - & $\ldots 27$ & $\emptyset$ & & $14-1$ & $5: 28-19$ \\
\hline 20 & - & - & $\ldots 20$ & 0 & & $14-1$ & 6 \\
\hline (9) & $(20)$ & $=$ & $\ldots 27$ & $\mathbf{1 0 - 2 7} \quad 11-2612-$ & $25 \quad 13-24$ & $14-1$ & $1: 9-20$ \\
\hline
\end{tabular}

Table 3. Execution for an objet with cavities 
The detection of a cavity results in a recursive call of $f$ illing () on the new interior contour ( $i_{19}$ et $i_{24}$, case $2 ; 9 ; i_{28}$, case 4 ). A call to fonction step_back () then manages poping linels up to discovery $\rightarrow$ begin included $\left(i_{2}, i_{3}\right)$. After incrementation of the contour index, a recursion on discovery->end takes place $\left(i_{3}, i_{4}, l_{21}\right.$ et $l_{33}$ table 3). Back from a recursive call, the current linel is restored to discovery->begin $\left(i_{5}\right.$, current between parentheses in the tables, $l_{(29)}$ table 3$)$.

Furthermore, after recursion $\left(i_{6}\right)$, and while explored is not empty, no span exploration happens and the fonction already_explored() accesses to the bottom of explored, whilst delist () dequeues $\left(i_{14}, i_{30}, l_{(9)}\right.$ table 3$)$. When explored gets exhausted, alrea dy_explored() returns the top of remainder, whilst delist () pops it (second $l_{18}$ table 3).

\section{Discussion}

In usual methods, and following the definition from [KR89], the border is a set of pixels. Following this border poses specific problems that Pavlidis collectively termed undersampling [Pav79]. We have considered a contour as a circular list of linels. Using a "border" in the common meaning of topology, undersampling problems have been disposed of.

In the general case, an exploration is saved in explored or remainder $\left(i_{25}, i_{26}\right.$, case 3$)$. A span which allows to claim that discovery actually leads to a new cavity is saved as well $\left(i_{18}, i_{23}\right)$, except in the case when its end is an interior linel. For that kind of relatively uncommon configuration (see span 8 in figure $4 \mathrm{~d}$ ), the span will be explored twice. Most of the spans are visited only once and the redundancy of binary map accesses is minor. The number of accesses to each pixel is thus in $O(n)$. This number is in $O(3 n)$ in Pavlidis's algorithm [Pav81]. In his approach, a span exploration systematically entails the one of the two neighboring spans, above and below, in order to determine the degree of this vertex in the line adjacency graph.

The number of recursive calls is proportional to the number of interior points in the naive version of the coloring algorithm. In usual "span" methods, among whom the one of Pavlidis, the size of the stack is in ratio to the number of spans in the boundary. In the proposed algorithm, the number of recursive calls is he number of cavities in the object. So the size of the stack is not a problem anyway.

The fact that the method is driven by contour marching directly gives the number of cavities. The approach preserves more topological information than usual methods, the latters being driven by the waiting structure for spans. This is attractive when having $3 \mathrm{D}$ applications in mind.

\section{$5 \quad$ Voxelized object surface traversal}

Given a cut plane, surfels of a voxelized object can be divided into two sets: slice surfels and ring surfels. The former are parallel to the cut plane and shaded in figure 5 . The latter take their name from the fact that in each slice, they can be described by circular lists. 

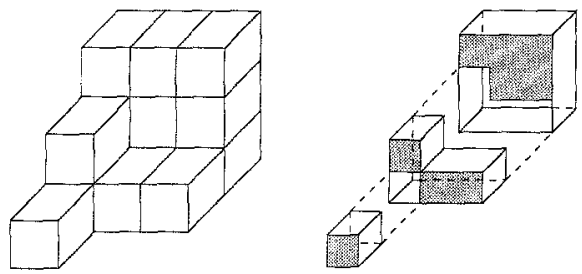

Fig. 5. Ring and slice surfels

Work is in progress to realize the surface tracking of a voxelized object according to a ring/slice scheme. It can be described informaly by the following procedure:

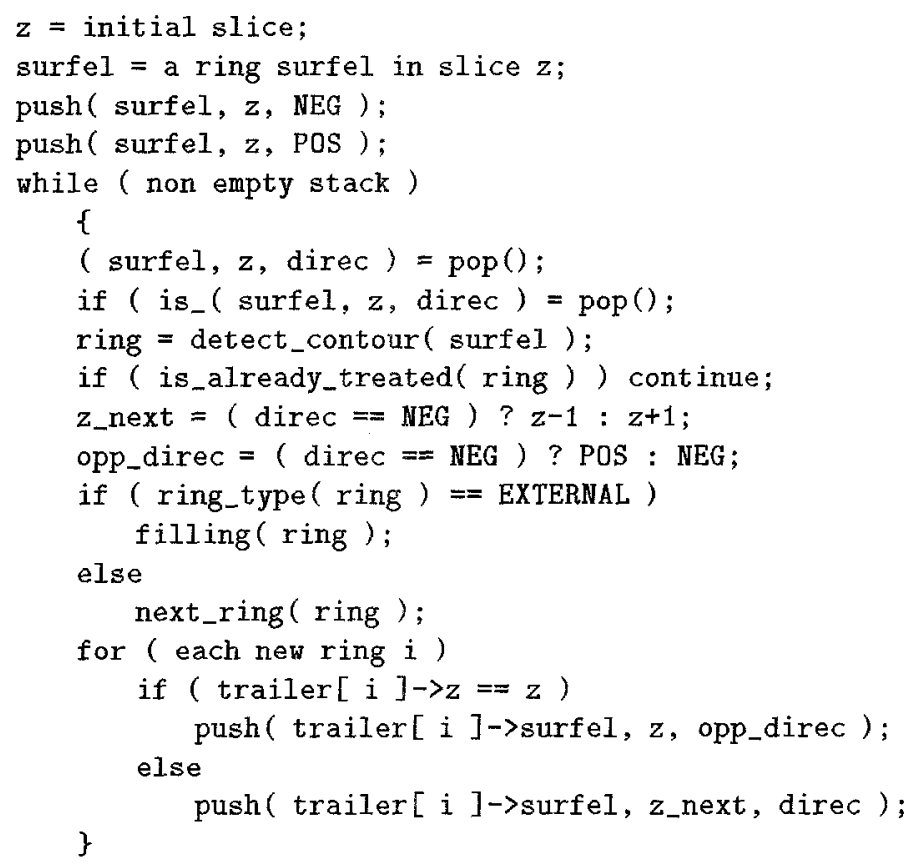

Fonction detect_contour ( ) is a contour tracking that chains detected surfels as a ring. The filling algorithm is adapted for the slice surfels detection. Fonction march_contour () is reduced to tracing the ring just built, whilst filling () is enriched with the management of a trailer table, indexed by interior contours. Each of its elements is a trailer surfel for an incoming ring, coupled up with the corresponding slice value. The latter is either the current ring slice value, or the one of a contiguous slice, depending on wether the ring type (external or internal) reverses or not.

The surface tracking method that is traditionnally used is the one described by Artzy et al. [AFH81]. Though elegant, the algorithm does not offer any a priori knowledge of the order in which surfels will be detected. This poses problems, especially pagination 
when accessing the 3D binary map [FHMU85]. It seems to be worthwhile to load in memory a few contiguous slices from the voxmap (we call it a "metaslice") and to treat it without having to access it again. Furthermore, if the detection progresses slice by slice, a parallel implementation can be taken under consideration, in which each processor should work on a different metaslice. Finally, this approach is also richer in topological information. It is indeed possible to build on the fly the Reeb graph of the object [SKK91].

More generally, this ring/slice scheme may be applied to every surfels graph traversal, for example the search for local extremums on the surface. It also directly leads to a $3 D$ filling algorithm.

\section{Conclusion}

We have proposed a $2 \mathrm{D}$ filling algorithm. It is free from undersampling problems because of its use of contours constituted of linels instead of pixels. The redundancy of binary map accesses is minor. The approach is recursive and the number of calls is only the number of cavities. The principle of a treatment of spans directed by contour marching directly provides the cavities. In that, it offers more topological information than usual techniques. The method finds an application in 3D voxelized objects surface traversal. It is in fact a basic procedure of algorithms for tracking, searching for extremum and filling of those objects surface.

The author wishes to thank the anonymous reviewers, D. Michelucci and B. Péroche for their constructive remarks on the preliminary version of this paper. 


\section{Appendix: pseudo-code}

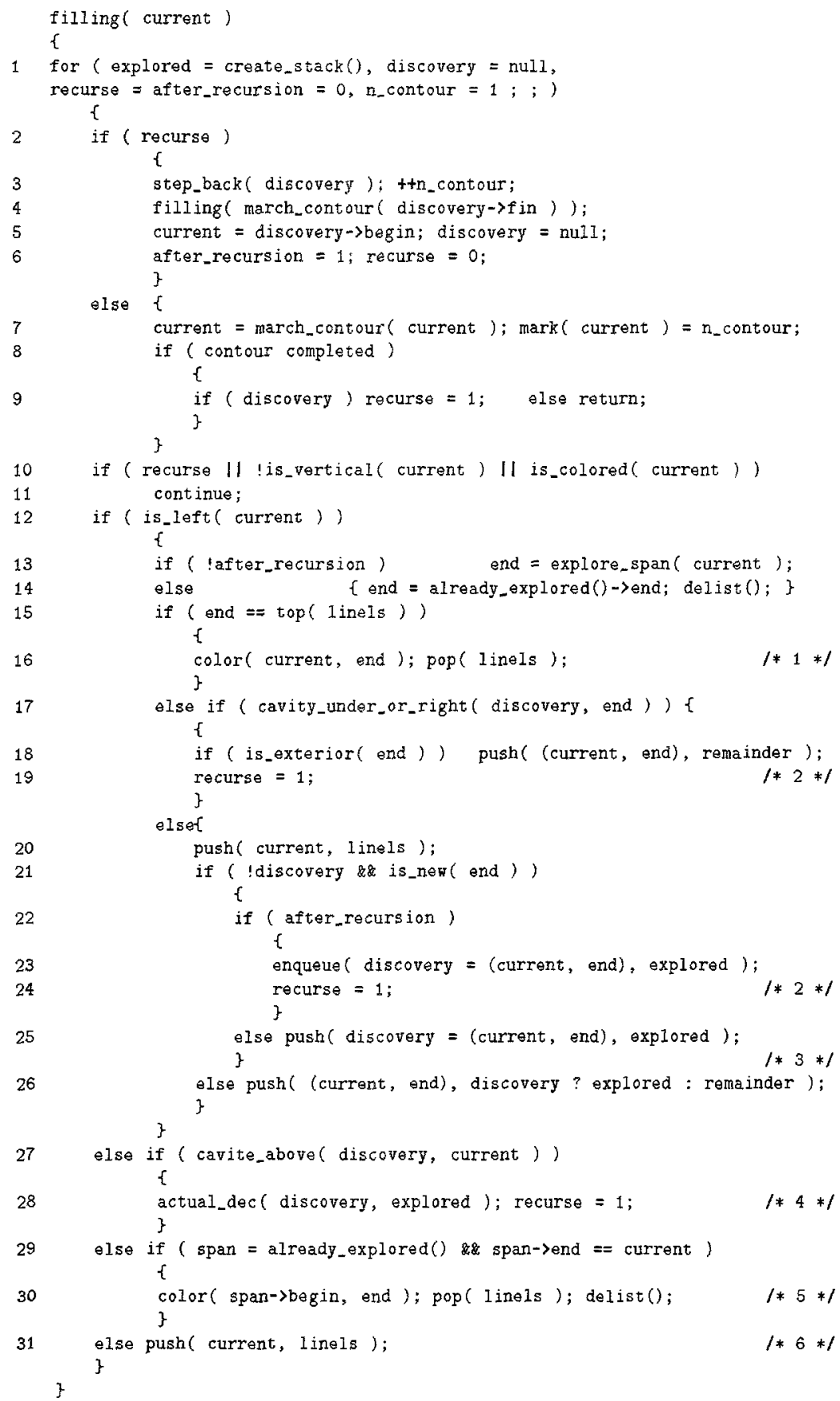




\section{References}

[AFH81] E. Artzy, G. Frieder, and G. Herman. The theory, design, implementation and evaluation of a three-dimensional surface detection algorithm. Computer graphics and image processing, (15):1-24, 1981.

[CM91] J.M. Chassery and A. Montanvert. Géométrie discrète en analyse d'images. Hermes, 1991.

[FHMU85] G. Frieder, G. Herman, C. Meyer, and J. Udupa. Large software problems for small computers: an example from medical imaging. IEEE Software, (2):37-47, September 1985 .

[Fra95] J. Françon. Topologie de khalimski-kovalevsky et algorithmique graphique. Technique et science informatiques, 14(10):1195-1219, 1995.

[FVFH90] Foley, VanDam, Feiner, and Hughes. Computer graphics: principles and practice. Addison Wesley, 2 edition, 1990.

[Heg85] G. Hegron. Synthèse d'image: algorithmes élémentaires. AFCET Dunod, 1985.

[KR89] T. Kong and A. Rosenfeld. Digital topology: introduction and survey. Computer vision, graphics and image processing, (48):357-393, 1989.

[Lie78] H. Lieberman. How to color in a coloring book. In SIGGRAPH'78, volume 12(3), pages 111-116. ACM, August 1978.

[Mat95] G. Mathieu. Points significatifs dans un contour discret : caractérisation des cavités et dilatation. In 5 th Discrete geometry for computer imagery, pages 249-258, 1995.

[MF82] D. Montuno and A. Fournier. Finding the $x-y$ convex hull of a set of $x-y$ polygons. Technical Report CSRG-148, University of Toronto, November 1982.

[Pav79] T. Pavlidis. Filling algorithms for raster graphics. Computer graphics and image processing, 10:126-141, 1979.

[Pav81] T. Pavlidis. Contour filling in raster graphics. In Proceedings SIGGRAPH'81, volume 15(3), pages 29-36. ACM, aug 1981.

[Sha80] U. Shani. Filling regions in binary raster images: a graph-theoretic approach. In SIGGRAPH'80, volume 14, pages 321-327. ACM, 1980.

[SKK91] Y. Shinagawa, T. Kunii, and Y. Kergosien. Surface coding based on morse theory. IEEE computer graphics and applications, 11(5):66-78, September 1991.

[Smi79] A. Smith. Tint fill. In SIGGRAPH'79, volume 13(2), pages 276-283. ACM, 1979.

[TL88] G. Tang and B. Lien. Region filling with the use of the discrete green theorem. Computer vision, graphics and image processing, (42):297-305, 1988. 